\title{
FERMION PRODUCTION IN STRONG MAGNETIC FIELD AND ITS ASTROPHYSICAL IMPLICATIONS
}

\author{
Hyun Kyu Lee* and Yongsung Yoon ${ }^{\dagger}$ \\ Department of Physics, Hanyang University, Seoul 133-791, Korea
}

\begin{abstract}
We calculate the effective potential of a strong magnetic field induced by fermions with anomalous magnetic moments which couple to the electromagnetic field in the form of the Pauli interaction. For a uniform magnetic field, we find the explicit form of the effective potential. It is found that the non-vanishing imaginary part develops for a magnetic field stronger than a critical field and has a quartic form which is quite different from the exponential form of the Schwinger process. We also consider a linear magnetic field configuration as an example of inhomogeneous magnetic fields. We find that the imaginary part of the effective potential is nonzero even below the critical field and shows an exponentially decreasing behavior with respect to the inverse of the magnetic field gradient, which is the non-perturbative characteristics analogous to the Schwinger process. These results imply the instability of the strong magnetic field to produce fermion pairs as a purely magnetic effect. The possible applications to the astrophysical phenomena with strong magnetic field are also discussed.

PACS numbers: $82.20 . \mathrm{Xr}, 13.40 . \mathrm{f}, 12.20 . \mathrm{Ds}$
\end{abstract}

Keywords: critical magnetic field, pauli interaction, anomalous magnetic moment, pair creation

\footnotetext{
*hyunkyu@hanyang.ac.kr

$\dagger$ cem@hanyang.ac.kr
} 


\section{INTRODUCTION}

Recent observations of the explosive phenomena like magnetars and gamma ray bursts indicate the possibility of very strong magnetic field around the compact objects [1, 2] which are considered to be responsible for powering the explosive events. The inferred field strength are larger than $\sim 10^{15} \mathrm{G}$, which is stronger than the conventional critical value $4 \times 10^{13} \mathrm{G}$. While most of the astrophysical environment is electrically neutral except the very narrow region near the surfaces of compact objects[3], the rotation of the magnetic field induces an electric field $\sim v_{\text {rotation }} B$. For $B \sim 10^{15} \mathrm{G}$, the induced electric field is estimated to be order of $\sim 10^{17} \mathrm{~V} / \mathrm{cm}$. Apparently the induced electric field strength is much larger than the critical field strength for electro-positron pair creation via Schwinger process [4]. However the invariant $B^{2}-E^{2}>0$ for this configuration indicates that the electromagnetic field configuration is dominated by the magnetic field. It has been known that the production of the minimally interacting fermion is not possible in the magnetically dominated configuration [5]. The pair production of minimally interacting particles is a purely electric effect. Hence the strong magnetic field configuration in the astrophysical environment is considered to be stable against the particle creation[6].

While the minimal coupling derived by the local gauge invariance is of fundamental nature, there appear also non-minimal couplings as well in the form of effective theory. Pauli introduced a non-minimal coupling of spin-1/2 particles with electromagnetic fields, which can be interpreted as an effective interaction of fermions with an anomalous magnetic moment [7, 8, 9]. For the neutral fermions with non-vanishing magnetic moments, it is the Pauli interaction through which the electromagnetic interaction can be probed. It is interesting to note that the inhomogeneity of the magnetic field, which couples directly to the magnetic dipole moment through Pauli interaction, plays a similar role analogous to the electric field for charged particles with the minimal coupling. The possibility of production of the neutral fermions in a purely magnetic field configuration with spatial inhomogeneity has been demonstrated in $2+1$ dimension[10], and the production rate in $3+1$ dimension has been calculated explicitly for the magnetic field with a spatial inhomogeneity of a critical value[11]. Recently the possibility of particle creations even in the uniform magnetic field[12] has been demonstrated provided the field strength is stronger than the critical value, which is the ratio of the fermion mass to its anomalous magnetic moment. This implies that 
the magnetic field configuration becomes unstable and the pair creation is possible as a purely magnetic effect. In section 2, the Pauli interaction is introduced and discussed in comparison with the minimal coupling. The details of the calculations of effective potentials for the uniform and the linear magnetic field configuration will be discussed in section 3 and 4 respectively. The results are summarized and their astrophysical implications are discussed in section 5 .

\section{PAULI INTERACTION}

The Dirac Lagrangian of a neutral fermion with Pauli interaction is given by

$$
\mathcal{L}=\bar{\psi}\left(\not p+\frac{\mu}{2} \sigma^{\mu \nu} F_{\mu \nu}-m\right) \psi
$$

where $\sigma^{\mu \nu}=\frac{i}{2}\left[\gamma^{\mu}, \gamma^{\nu}\right], \quad g_{\mu \nu}=(+,-,-,-) . \mu$ in the Pauli term measures the magnitude of the magnetic moment of fermion. The corresponding Hamiltonian is given by

$$
H_{\text {pauli }}=\vec{\alpha} \cdot(\vec{p}-i \mu \beta \vec{E})+\beta(m-\mu \vec{\sigma} \cdot \vec{B})
$$

where $\sigma^{i}=\frac{1}{2} \epsilon^{i j k} \sigma^{j k}$. It is also known that the Hamiltonian for a charged fermion with minimal coupling is given by

$$
H_{\text {dirac }}=\vec{\alpha} \cdot(\vec{p}-e \vec{A})+\beta(m-\beta e \phi)
$$

One can note a kind of duality between $\vec{B}$ of Pauli Hamiltonian and $\phi$ of Dirac Hamiltonian modulo $\gamma$ matrices. Let us consider the Pauli Hamiltonian with pure magnetic field, $\vec{E}=0$, and the Dirac Hamiltonian with pure electric field, $\vec{A}=0$. Then, for a static limit, $\vec{p}=0$, we get

$$
H_{\text {pauli }} \rightarrow \beta(m-\mu \vec{\sigma} \cdot \vec{B}), \quad H_{\text {dirac }} \rightarrow \beta(m-\beta e \phi) .
$$

In this simple static limit, a particle state is considered to be assigned with $\beta=+1$ and antiparticle state with $\beta=-1$. Then the energy difference between the particle and antiparticle state is given by

$$
\Delta H_{\text {pauli }} \rightarrow 2(m-\mu \vec{\sigma} \cdot \vec{B})
$$

for the Pauli interaction and

$$
\Delta H_{\text {dirac }} \rightarrow 2 m
$$


for the minimal interaction. One can see that there is a level crossing with Pauli interaction for a strong enough magnetic field. For a uniform magnetic field, the energy eigenvalues of the Hamiltonian Eq.(2) are given by

$$
E= \pm \sqrt{p_{l}^{2}+\left(\sqrt{m^{2}+p_{t}^{2}} \pm \mu B\right)^{2}}
$$

where $p_{l}$ and $p_{t}$ are respectively the longitudinal and the transversal momentum to the magnetic field direction. One can see that for a magnetic field stronger than the critical field $B_{c}=\frac{m}{\mu}$, the ground state with $p_{l}=p_{t}=0$ crosses zero energy state. Theoretically the particle creation is known to be associated with level crossing[13]. Analogously in this example one can easily guess the pair creation for $B>B_{c}$ : the state occupied the negative energy sea becomes a particle state and the vacant positive energy state plunges into the negative sea to make an antiparticle state. This indicates the possible instability of the magnetic field configuration. In section 3, we will show explicitly that the imaginary part of the effective potential develops exactly when $B=B_{c}$. On the other hand, the energy eigenvalues of minimally interacting charged fermions are

$$
E= \pm \sqrt{p_{l}^{2}+m^{2}+|e| B(2 n+1-\operatorname{sgn}(e) \hat{s})},
$$

where $n=0,1,2, \ldots$, and $\hat{s}= \pm 1$ is the spin projection along the magnetic field[14]. It should be pointed out that the ground state energy is independent of the external magnetic field and moreover no state plunges into the zero energy state even for a strong magnetic field. The observation that particle production of minimally interacting fermions is impossible in pure magnetic fields can be attributed to this finite energy gap.

However, for a charged particle with minimal coupling, this energy gap, $2 m$, can be overcome by the virtual work done by the electric field up to the Compton wave length. This process has been known as Schwinger process [4] with a pair creation rate similar to the tunnelling process[15]. The approximate duality between $\vec{B}$ of Pauli Hamiltonian and $\phi$ of Dirac Hamiltonian indicates that the inhomogeneity of the magnetic field manifested in the form of $\vec{\nabla} \times \vec{B}$, which couples directly to the magnetic dipole moment through Pauli interaction, plays a similar role analogous to the electric field for a charged particle with the minimal coupling. It is interesting to note that this duality is exact in $2+1$ dimension[10]. Hence we can expect fermion pair creation even for $B<B_{c}$. Denoting the spatial derivative as $\left|B^{\prime}\right|$, we can expect the particle creation for strong enough magnetic field such that the 
virtual work along the Compton wavelength, $\lambda=1 / m$, is comparable to the mass:

$$
\mu\left|B^{\prime}\right| \lambda \sim m
$$

In analogy to the Schwinger process the production rate is expected to have a following form:

$$
w \sim m^{4} \exp ^{- \text {constant } \times m^{2} /\left|\mu B^{\prime}\right|}
$$

The details of the calculation of effective potential for the linear magnetic field configuration will be discussed in section 4 .

\section{EFFECTIVE POTENTIAL FOR THE UNIFORM MAGNETIC FIELD}

In general, the effective potential, $V_{\text {eff }}(A)$, for a background electromagnetic vector potential, $A_{\mu}$, can be obtained by integrating out the fermion:

$$
-i \int d^{4} x V_{\mathrm{eff}}(A[x])=\int d^{4} x<x\left|\operatorname{tr} \ln \left\{\left(\not p+\frac{\mu}{2} \sigma^{\mu \nu} F_{\mu \nu}-m\right) \frac{1}{\not p-m}\right\}\right| x>,
$$

where $F_{\mu \nu}=\partial_{\mu} A_{\nu}-\partial_{\nu} A_{\mu}$, and $t r$ denotes the trace over Dirac algebra. The decay probability of the background magnetic field into the neutral fermions is related to the imaginary part of the effective potential $V_{\text {eff }}(A)$,

$$
P=1-\left|e^{i \int d^{4} x V_{\text {eff }}(A[x])}\right|^{2}=1-e^{-2 \Im \int d^{3} x d t V_{\text {eff }}(A[x])} .
$$

That is, the twice of the imaginary part of the effective potential $V_{\text {eff }}(A[x])$ is the fermion production rate per unit volume[15]: $w(x)=2 \Im\left(V_{\text {eff }}(A[x])\right)$ for small probabilities.

For a uniform magnetic field configuration such that $\vec{B}=B \hat{z}$, the integral form of the effective potential is obtained as[11]

$$
V_{\mathrm{eff}}=-\frac{(\mu B)^{2}}{4 \pi^{2}} \int_{0}^{\infty} \frac{d s}{s^{2}}\left[i \int_{0}^{1} d \xi(1-\xi) e^{i(\mu B)^{2} \xi^{2} s}-\frac{i}{2}+\frac{(\mu B)^{2} s}{12}\right] e^{-i m^{2} s} .
$$

This integration can be done explicitly. Introducing dimensionless parameters, $t=m^{2} s$ and $\beta=\frac{|\mu B|}{m}$, the imaginary part of the effective potential Eq.(13) can be written as

$$
\Im\left(V_{\text {eff }}\right)=-\frac{m^{4} \beta^{2}}{8 \pi} \int_{0}^{1} d \xi(1-\xi)\left[1-\beta^{2} \xi^{2}-\left|1-\beta^{2} \xi^{2}\right|\right] .
$$

One can easily see that for a magnetic field weaker than the critical field, $\beta \leq 1$, the integration Eq.(14) vanishes. It can be also verified by a contour integration[11]. Therefore, 
one can see that the uniform magnetic fields weaker than the critical field are stable as expected.

However, for a magnetic field stronger than the critical field, $\beta>1$, the imaginary part of the effective potential does not vanish, but gives a positive value:

$$
\Im\left(V_{\text {eff }}\right)=\frac{1}{48 \pi}(|\mu B|-m)^{3}(|\mu B|+3 m) .
$$

This is exactly what is expected that the pair creation is associated with the level crossing of positive and negative energy states. It is interesting to note that the development of the imaginary part associated with a level crossing has been also demonstrated in the different contexts, for example in the work of Graham and Jaffe[13]. Therefore, the uniform magnetic fields stronger than the critical field are unstable, and reduce their strengths producing the fermion pairs. This implies that there is an upper bound for stable magnetic field strengths determined by the particle whose ratio of the mass to the magnetic moment, $\frac{m}{\mu}$, is the least among the fermions which couple to electromagnetic fields through the Pauli interaction. Pauli interaction in $2+1$ dimension shows quite different physics compared that in $3+1$ dimension. The development of the non-vanishing imaginary part of the effective potential for uniform magnetic fields stronger that the critical field strength does not happen in $2+1$ dimension. It is because the energy gap between the particle and the antiparticle states is $2 m$, which is independent of magnetic field strength even in the presence of the Pauli interaction in $2+1$ dimension.

For the completeness, the real part of the effective potential Eq.(13) has been also calculated explicitly as shown in FIG. 1. For a weak field, $\beta \ll 1, \Re\left(V_{\text {eff }}\right)$ approximates to $\frac{(\mu B)^{6}}{240 \pi^{2} m^{2}}$, and for the critical field, $\beta=1, \Re\left(V_{\text {eff }}\right)=(96 \ln 2-65) \frac{m^{4}}{288 \pi^{2}}$. The real and imaginary parts of the effective potential with respect to the magnetic field strength are shown together in FIG.1 in the unit of $\frac{m^{4}}{48 \pi^{2}}$.

It is also interesting to see how the instability due to the Pauli interaction is affected when the minimal coupling is turned on in addition to the Pauli interaction. Let us consider an effective Lagrangian, which might describe a fermion endowed with a non-vanishing electric charge $e$ and as well as with a magnetic dipole moment $\mu$, given by

$$
\mathcal{L}=\bar{\psi}\left(\not p-e \not A+\frac{\mu}{2} \sigma^{\mu \nu} F_{\mu \nu}-m\right) \psi .
$$

In this work, for the simplicity we consider the electric charge $e$ and the anomalous magnetic dipole moment $\mu$ as two independent couplings. It is straightforward to show that the 


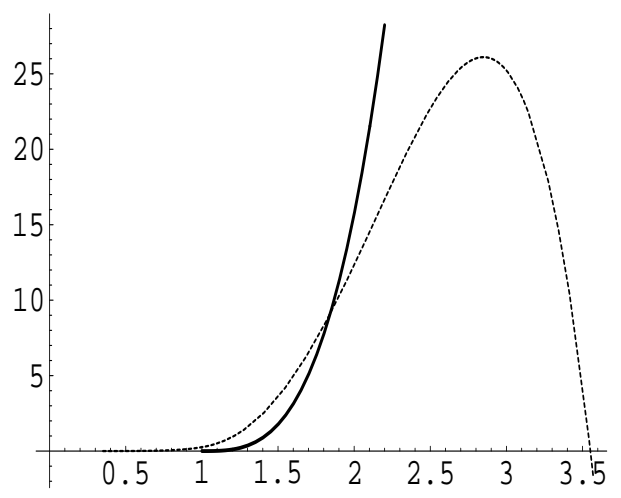

FIG. 1: Effective potential of the uniform magnetic field B for neutral fermions with a magnetic moment: vertical axis is $V_{\text {eff }}$ in the unit of $\frac{m^{4}}{48 \pi^{2}}$ (the solid line is for the imaginary part and the dashed line is for the real part), horizontal axis is $\beta(=|\mu B| / m)$.

imaginary part is not affected by the addition of the independent minimal coupling [12]. Hence we can see that for the fermion described by Eq.(16) the minimal coupling does not affect the instability due to the magnetic moment through the Pauli interaction.

In QED, however, $e$ and $\mu$ are not independent parameters. $\mu$ could be identified as the Schwinger's anomalous magnetic moment $\mu_{a}=\frac{\alpha}{2 \pi} \frac{e}{2 m}$, which comes from the 1-loop radiative corrections [15]. The full calculation of the QED radiative correction for strong magnetic fields 16] shows that the Pauli term description of the Schwinger's anomalous magnetic moment is valid only for weak magnetic fields such that $B \ll m^{2} / e$, which is much smaller than the critical field for the anomalous magnetic moment defined by $B_{c}=m / \mu_{a}$. It is found that there is no level crossing associated with the pair creation and the pure magnetic configuration is stable with minimally interacting charged fermions $[6]$.

\section{PRODUCTION RATE FOR A LINEAR MAGNETIC FIELD}

In the previous section, we observe that the anomalous magnetic moment induces an instability of uniform magnetic field for $B \geq B_{c}$. However, the instability due to the anomalous magnetic moment even for $B \leq B_{c}$ occurs provided that the field gradient $B^{\prime}$ is strong enough to overcome the energy gap as discussed in Section 2.

We consider a static linear magnetic field configuration with a constant gradient along an orthogonal direction to the magnetic field in $3+1$ dimension[11]. We take $\hat{z}$-direction as the 
magnetic field direction with a constant gradient along $\hat{x}$-direction, $\vec{B}=B(x) \hat{z}$, such that

$$
F_{12}=B(x)=B_{0}+B^{\prime} x=B^{\prime} \widetilde{x}, \quad\left(\widetilde{x}=x_{*}+x, \quad x_{*}=\frac{B_{0}}{B^{\prime}}\right),
$$

where the field gradient $B^{\prime}$ is a non-zero constant. After lengthy but straightforward calculations we get the effective potential given by

$$
\begin{aligned}
V_{\mathrm{eff}}= & -\frac{(\mu B)^{2}}{4 \pi^{2}} \int_{0}^{\infty} \frac{d s}{s^{2}}\left\{i \sqrt{\mu B^{\prime} s \operatorname{coth}\left(\mu B^{\prime} s\right)} \int_{0}^{1} d \xi(1-\xi) e^{i \frac{(\mu B)^{2}}{\mu B^{\prime}} \xi^{2} \tanh \left(\mu B^{\prime} s\right)}-\frac{i}{2}\right. \\
& \left.+\frac{(\mu B)^{2} s}{12}\right\} e^{-i m^{2} s}+\frac{1}{8 \pi^{2}} \int_{0}^{\infty} \frac{d s}{s^{3}}\left\{\left(\mu B^{\prime} s \operatorname{coth}\left(\mu B^{\prime} s\right)\right)^{3 / 2}-1-\frac{\left(\mu B^{\prime} s\right)^{2}}{2}\right\} e^{-i m^{2} s} .
\end{aligned}
$$

The divergent contributions at $s=0$ are removed by adding local counter terms of $(\mu B)^{2}$, and $(\mu B)^{4}$ in the first integral. This implies the renormalization of the magnetic moment $\mu$ to the measured value and the coupling of $(\mu B)^{4}$ to zero presumably. An additional divergent contribution at $s=0$ is removed by adding a local counter term of $\left(\mu B^{\prime}\right)^{2}$ in the second integral.

The effective potential for the uniform field configuration Eq.(13) can be also obtained by putting $\mu B^{\prime}=0$ in Eq.(18).

Introducing dimensionless parameters defined as $t=s m^{2}$ and $\beta^{\prime}=\frac{\left|\mu B^{\prime}\right|}{m^{2}}$, the production rate density $w(x)$ in the unit of the fermion mass is finally given by

$$
\begin{aligned}
w(x)= & -\frac{2 m^{4}}{4 \pi^{2} \lambda \kappa} \int_{0}^{\infty} \frac{d v}{v^{2}}\left\{\sqrt{v \operatorname{coth} v} F\left(\frac{\lambda}{\kappa} \tanh v, \lambda v\right)-\frac{1}{2} \cos \lambda v-\frac{\lambda v}{12 \kappa} \sin \lambda v\right\} \\
& -\frac{m^{4}}{4 \pi^{2} \lambda^{2}} \int_{0}^{\infty} \frac{d v}{v^{3}}\left\{(v \operatorname{coth} v)^{3 / 2}-1-\frac{v^{2}}{2}\right\} \sin \lambda v,
\end{aligned}
$$

where

$$
F(a, b) \equiv \int_{0}^{1} d \xi(1-\xi) \cos \left(a \xi^{2}-b\right)
$$

For a linear magnetic field configuration, the magnetic field variation at the scale of the Compton wavelength of the fermion is $\left|B^{\prime}\right| / m$. Therefore, the background magnetic field weaker than $\left|B^{\prime}\right| / m$ is unphysical. That is, the spatial gradient of the background magnetic field $\left|B^{\prime}\right|$ should be smaller enough than $m|B|, \beta^{\prime}<\beta$. Since the integration Eq.(19) has essential singularities along the imaginary axis, we have not succeeded to get an analytic expression of the integrations in Eqs.(18),19), but can obtain the numerical results, in which the the physical requirement, $\beta^{\prime}<\beta$, turns out to be the necessary condition to make the production rate density $w(x)$ positive. 
As seen in FIG.2 and FIG.3, provided that the field gradient is extremely large, fermion pair creation is possible for a magnetic field weaker than the critical field. The particle production rate shows an exponentially decreasing behavior with respect to the inverse of the field gradient for a fixed strength $|B|<B_{c}$ in the form of Eq.(10), which is the characteristics of the non-perturbative process. This can be understood as a quantum tunnelling through an energy gap $\sim 2(m-\mu B)$ of a particle with the help of quantum energy fluctuation $\sim \mu\left|B^{\prime}\right| / m$ due to the inhomogeneous magnetic field coupled to the anomalous magnetic moment through Pauli interaction. This is similar to the Schwinger process of electron-positron pair creation overcoming the mass gap $2 m$ in the strong electric field with the help of quantum energy fluctuation $|e E| / m$, where the production rate is decreasing exponentially[15], $w \sim e^{-\operatorname{constant} \times m^{2} /|e E|}$.

However, for magnetic fields stronger than the critical field, it turns out that the spatial inhomogeneity of magnetic fields does not help fermion production, but reduces the production rate as seen in FIG.2. It is found that the production rate density Eq.(19) approaches to $\frac{m^{4}}{24 \pi}(\beta-1)^{3}(\beta+3)$, which is the production rate density for a uniform magnetic field from Eq.(15), as $\beta^{\prime} \rightarrow 0$.

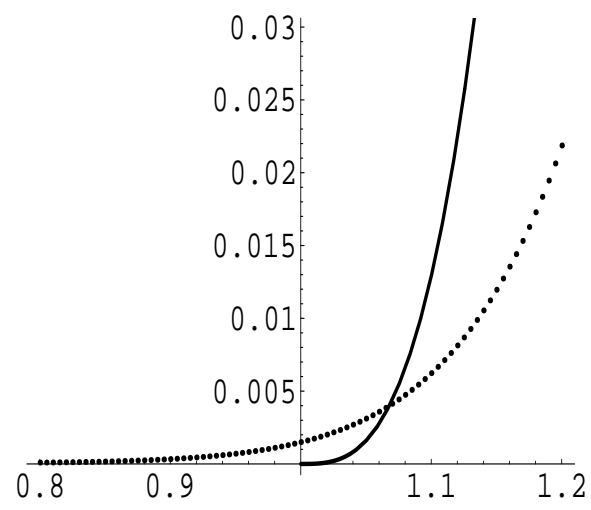

FIG. 2: Production rate density $w$ in the unit of $\frac{m^{4}}{4 \pi^{2}}$ for $\beta^{\prime}=0.1$ (dotted line) and $\beta^{\prime}=0$ (solid line) with varying $\beta$.

\section{DISCUSSION}

In this paper, we discuss the possibility of particle creation in the strong magnetic field as a magnetic effect in contrast to the Schwinger process for creating a charged pairs as an 


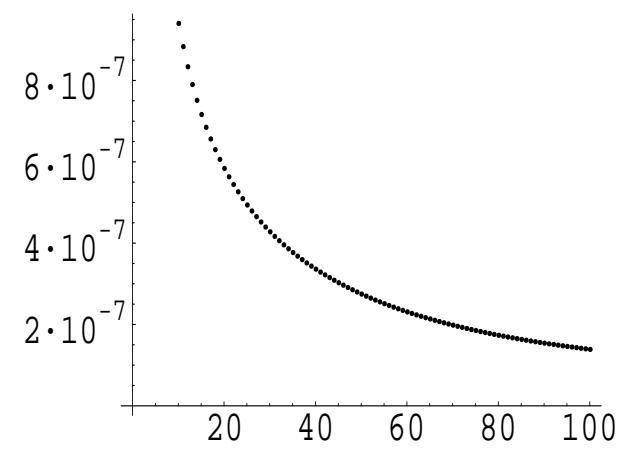

FIG. 3: Production rate density $w$ in the unit of $\frac{m^{4}}{4 \pi^{2}}$ for $\beta=0.1$ with varying $1 / \beta^{\prime}$.

electric effect. It has been well known that pair creation of minimally interacting fermions is not possible in magnetically dominant configurations [4, 5, 6]. However, it is found that in a purely magnetic field configuration the instability of the magnetic field also occurs due to the anomalous magnetic moment of spin-1/2 fermions through Pauli interaction[11, 12]. The instability induced by the neutral fermion with magnetic moment has been demonstrated explicitly by showing the emergence of imaginary parts of the effective potential either when the field strength becomes stronger than the critical field $B_{c}=\frac{m}{\mu}$ or when the gradient of the magnetic field is strong enough $B^{\prime} \sim m B_{c}$. For a uniform magnetic field $B \geq B_{c}$, we get a simple analytical form of the production rate density $w$ given by $\frac{m^{4}}{24 \pi}\left(\frac{|\mu B|}{m}-1\right)^{3}\left(\frac{|\mu B|}{m}+3\right)$. We also demonstrate that the production rate for the inhomogeneous magnetic field is of order $m^{4}$ for $B \sim B_{c}$. These results imply the instability of the strong magnetic field to produce fermion pairs as a purely magnetic effect.

Recent observations of the explosive astrophysical phenomena like Soft Gamma Repeaters(SGRs), Anomalous X-ray Pulsars(AXPs) [1] and Gamma Ray Bursts(GRBs) [2] indicate that the extraordinarily strong magnetic fields $\left(\geq 10^{15} \mathrm{G}\right)$ can be expected in the vicinity of the compact objects, neutron stars and black holes, which are supposed to be located at the center of the activities. Since the astrophysical environment is most likely neutral, the environments are considered to be magnetically dominant. It has been argued that the magnetized vacuum is stable against the electron-positron pair creation and the only instability might be due to the monopole pair creation near the Planck scale[6] which leads to the critical field strength $B_{c} \sim 10^{23}-10^{53} \mathrm{G}$, as far as particles with non-vanishing electric or magnetic charges are considered. However provided that there is a neutral fermion for 
which the interaction with an electromagnetic field is described by Pauli interaction, Eq.(11), it is very interesting to discuss whether the magnetic vacuum instability near the critical field might have any interesting implication on the astrophysical phenomena dominated by strong magnetic field.

As an example, neutrinos are electrically neutral but are known to have nonzero-mass from the neutrino oscillation observations[17]. So far there is no experimental evidence for the magnetic moment of the neutrinos but one can not simply rule out the magnetic moment just because of the electrical neutrality. In a minimal extension of the standard model[18] to incorporate the neutrino mass the magnetic moment of neutrino is calculated to be $\sim 10^{-20} \mu_{B}$ if we take the neutrino mass as a cosmological bound on the neutrino mass [19], $m_{\nu} \sim 10^{-1} \mathrm{eV}$. Beyond standard model calculations [20, 21, 22] yield also a wide range of neutrino magnetic moment up to the current laboratory upper limit[24], $\mu_{\nu}<10^{-10} \mu_{B}$. Then the lower limit of the critical field strength is given by $B_{c}=\mu_{\nu} / m_{\nu} \geq 10^{17} \mathrm{G}$ when we take the neutrino magnetic moment as a current upper limit. It is interesting to note that the critical field strength $B_{c} \sim 10^{17} \mathrm{G}$ is not so inconsistent with $10^{15} \mathrm{G}$ inferred from SGRs, AXPs and GRBs in the sense that it can be attained assuming a specific dynamo in the neutron star [23]. The production rate of the neutrino pair due to the magnetic vacuum instability for the critical field is proportional to $m_{\nu}^{4}$ and is given as $w \sim 10^{31} / \mathrm{m}^{3} \mathrm{~s}$. The neutrino luminosity can be estimated as

$$
L_{\nu} \sim w \times m_{\nu} \times R_{N S}^{3} \sim 10^{30} \mathrm{erg} / \mathrm{s} .
$$

However it turns out to be much smaller than the X-ray luminosity of magnetars, $L_{X} \sim$ $10^{35} \mathrm{erg} / \mathrm{s}$ such that any appreciable effects due to the magnetic vacuum instability via neutrino pair production may be hardly observed from the magnetars.

Basically for a particle with mass $m$ and magnetic moment $\mu$, the pair production rate is $\sim m^{4}$ near the critical field strength $B_{c}=m / \mu$. The characteristic time scale is given by $\tau_{B} \sim B_{c}^{2} / m^{5}$. With $R_{B}^{3}$ as an effective volume of the magnetosphere, the luminosity can be estimated as $L \sim m^{5} R_{B}^{3}$. Provided $B_{c} \sim B_{\text {magnetar }}$ and $R_{B} \sim 10^{4} \mathrm{~m}$ as those of a magnetar, a larger luminosity for observation can be easily obtained with a massive particle but the magnetic moment should be increasing to keep the critical field strength, which constrains significantly models for particles involved. Hence for an interesting observable effects due to the magnetic vacuum instability to be discussed there should be a physically well-motivated 
effective theory which admits the Pauli interaction for particles with mass and magnetic moment appropriate to the environment, perhaps most likely related to the astrophysical phenomena, which produces a strong magnetic field up to the critical field strength.

This work was supported by grant No. (R01-2006-000-10651-0) from the Basic Research Program of the Korea Science \& Engineering Foundation.

[1] P.M. Woods and C. Thompson, astro-ph/0406133(2004)

[2] H.K. Lee, R. Wijeres and G.E. Brown, Phys. Rep. 325, 83 (1999)

[3] V.V. Usov, Phys. Rev. Lett. 80, 230(1998)

[4] J. Schwinger, Phys. Rev. 82, 664(1951)

[5] G. Dunne and T.M. Hall, Phys. Lett. B419, 322(1998); Phys. Rev. D60, 065002(1999); S.P. Kim and D. Page, Phys.Rev. D73 (2006) 065020

[6] R. C. Duncan, astro-ph/0002442

[7] W. Pauli, Rev. Mod. Phys. 13, 203(1941)

[8] P.M. Lavrov, J. Phys. A18, 3455(1985); W. Dittrich and M. Reuter, Lect. Notes Phys. 220, $1(1985)$

[9] C-L. Ho and P. Roy, Annals. Phys.(NY) 312, 161(2004); Q.-G. Lin, Phys. Rev. A61, 022101(2000)

[10] Q-G Lin, J. Phys. G. 25, 1793(1999)

[11] H.K. Lee and Y. Yoon, JHEP 0603, 78(2006)

[12] H.K. Lee and Y. Yoon, JHEP 0703, 86(2007)

[13] N. Graham and R.L. Jaffe, Phys.Lett. B435, 145 (1998)

[14] V.R. Khalilov, Phys. Rev. D65, 056001(2002)

[15] C. Itzykson and J.B. Zuber, Quantum Field Theory, McGraw-Hill, New York, 1980

[16] B. Jancovici, Phys. Rev. 187, 2275(1969); W.-y. Tsai and A. Yildiz, Phys. Rev. D8, 3446(1973);

R. Geprägs, H. Riffert, H. Herold, H. Ruder, and G. Wunner, Phys. Rev. D49, 5582(1994).

[17] M. Maltoni, T. Schwetz, M. A. Tortola, and J. W. F. Valle, New J. Phys. 6 (2004) 122;G. L.

Fogli, E. Lisi, A. Marrone, and A. Palazzo, Prog. Part. Nucl. Phys. 57 (2006) 742

[18] K. Fuzikawa and R. Shrock, Phys. Rev. Lett. 45, 963(1980)

[19] J. Lesgourgues and S. Pastor, Phys. Rept. 429 (2006) 307 
[20] G.C. McLaughlin and J. N. Ng, Phys. Lett. B470, 157(1999).

[21] C.-K, Chua and W.-Y. P. Hwang, Phys. Rev. D60, 073002(1999)

[22] N. F. Bell, M. Gorchtein, J. Ramsey-Musolf, P.Vogel and P.Wang, astro-ph/0606248

[23] C. Thomson and R.C. Duncan, Astrophys. J. 408, 194(1993)

[24] D. W. Liu et al., Phys. Rev. Lett. 93, 021802(2004); Z. Darakchieva et al., Phys. Lett. B 615, $153(2005)$ 